\title{
Microplastics have shape- and polymer- dependent effects on soil aggregation and organic matter loss - an experimental and meta-analytical approach
}

\author{
Anika Lehmann ${ }^{1,2^{*}}$ D, Eva F. Leifheit ${ }^{1,2}$, Maurice Gerdawischke ${ }^{1}$ and Matthias C. Rillig ${ }^{1,2}$
}

\begin{abstract}
Microplastics are a diverse and ubiquitous contaminant, a global change driver with potential to alter ecosystem properties and processes. Microplastic-induced effects in soils are manifold as microplastics differ in a variety of properties among which the shape is of special interest. Our knowledge is limited regarding the impact of various microplastic shapes on soil processes. Therefore, we conducted this two-part research comprising a meta-analysis on published literature and a lab experiment focusing on microplastic shapes- and polymer-induced effects on soil aggregation and organic matter decomposition. We here focus on fibers, films, foams and particles as microplastic shapes.

In the meta-analysis, we found a strong research focus on fibrous and particulate microplastic materials, with films and foams neglected.

Our experiment showed that microplastic shapes are important modulators of responses in soil aggregation and organic matter decomposition. Fibers, irrespective of their chemistry, negatively affected the formation of aggregates. However, for other shapes like foams and particles, the polymer identity is an important factor comodulating the soil responses.

Further research is needed to generate a data-driven foundation to permit a better mechanistic understanding of the importance and consequences of microplastics added to soils.
\end{abstract}

Keywords: Microplastic, Soil aggregation, Organic matter loss, Shape, Experiment, Meta-analysis

\section{Introduction}

Microplastics are proposed as a new global change driver with increasingly realized impacts on marine, aquatic and terrestrial systems [1-5]. Microplasticinduced effects on ecosystem functions and processes are starting to be revealed, as research shifts from a more ecotoxicological focus to more fully embrace an ecosystem perspective $[5,6]$.

\footnotetext{
* Correspondence: lehmann.anika@googlemail.com

${ }^{1}$ Freie Universität Berlin, Institut für Biologie, 14195 Berlin, Germany ${ }^{2}$ Berlin-Brandenburg Institute of Advanced Biodiversity Research, 14195 Berlin, Germany
}

\section{Springer Open}

Microplastics are not a monolithic issue and neither are the effects they induce. Microplastics are a group of synthetic polymers encompassing a wide diversity in sizes, shapes, chemical composition and additives $[7,8]$. They are either produced as primary microplastics or secondarily via degradation in the environment to fragments $<5 \mathrm{~mm}$. Plastic particles are exposed to weathering and degradation in the environment causing further fragmentation and leaching of additives [9-11].

Our knowledge about the effects of microplastics on soils is still limited, although they likely are exposed to microplastic globally and at increasing levels, especially agricultural and urban soils. Microplastics enter soils via 
atmospheric deposition [12, 13], plastic mulching [14], sewage sludge [15] and compost [16]. Microplastics are ubiquitous and persistent $[8,17]$. After entering the soil matrix they have the potential to alter soil properties and processes, e.g. bulk density, water holding capacity and soil aggregate stability [18].

Microplastics can affect soils as a function of their chemistry, but also through their shape [19]. Microplastics can resemble natural shapes, or they can be quite dissimilar from naturally-occurring objects in soil. In soils, microplastic beads and particles (non-spherical, irregular shape) can be almost indistinguishable from sand grains, for example. Microplastic fibers (rigid and unbranched, thread-like filaments) diverge clearly from hyphae, plant roots or other natural filamentous, linear organisms or structures. Other microplastic shapes like film or foil particles (planar and rigid shape) and foams (sponge-like, porous, irregular shape) have no analogs in a natural system due to their properties and size. Research on terrestrial systems revealed that a key feature of soil - its structural stability - is often negatively affected by microplastic fibers, while for microplastic beads and particles there is a range of positive to negative effects (e.g. [18, 20-22]). The spatial arrangement of primary particles into aggregates, and the resulting pore networks, together constitute the soil structure $[23,24]$ and strongly control soil processes, including biogeochemical cycles and soil carbon storage and processing $[25,26]$. For example, soil organic matter can be physically protected inside of soil aggregates. However, the detrimental microplastic-induced effects on soil structure and microbial activity are suggested to indirectly diminish this protective ability of soil aggregates [18, 20, 27]; however, the observed effects are variable, shifting with microplastic concentration and properties. To better understand effects of microplastics on soil aggregation and organic matter processing, it is thus necessary to more systematically examine key microplastic properties, for example their shape.

To this end, we here present a two-part study, consisting of a quantitative synthesis based on available data, and a laboratory experiment systematically varying microplastic shapes. Research on microplastic-induced effects on soil systems is accumulating and with it the need for a quantitative evaluation of the consequences for soil structure and soil organic matter loss. Hence, we here conducted a meta-analysis on published articles presenting microplastic-induced effects on soil aggregation and soil organic matter loss. We identified overall 10 articles for which we extracted data on soil aggregate formation, stability and soil organic matter loss and information on microplastic shape and polymer. With the resulting datasets we investigated (1) knowledge clusters and gaps for microplastic shape and polymer effects on soil aggregate formation and stability and soil organic matter loss. For the knowledge clusters we evaluated (2) the overall impact of microplastic soil contamination on soil aggregate formation and stability and soil organic matter loss and (3) how these effects are modulated by microplastic shape and polymer. The identified knowledge gaps highlighted the need to add new insights on the importance of microplastic shapes beyond fibers and particles. Therefore, we designed a laboratory study in which we tested the effect of four different shapes, each represented by three different polymers, on soil aggregation and organic matter decomposition. Based on the findings from our meta-analysis we hypothesize (1) that fibers, films, foams and particles have distinct impacts on soil structure and organic matter loss; and (2) that the shape is more important than the polymer.

\section{Material and methods Meta-analysis}

First, we conducted a meta-analysis on published articles reporting microplastic effects on soil aggregation (i.e. soil aggregate formation and stabilization) and/ or organic matter loss. We followed the PRISMA guidelines [28] (for further information see supplementary information; SI Fig. S1).

\section{Literature search}

We conducted a two-step literature search on 8th of September 2020 to identify and collect articles for our database. First, we used the search string (TS $=(($ microplastic* OR microplastic*) NOT (marine OR sea\$ OR river" OR coast\$ OR coastal OR "freshwater" OR "freshwater" OR beaches OR beach OR ocean" OR bay OR atlantic OR lake\$ OR pacific OR estuar* OR gulf OR seawater OR sediment\$ OR coral\$ OR "fish" OR daphnia OR mussel\$ OR zooplankton OR "Mytilus edulis" OR "bird OR "birds OR "Arenicola marina" OR "Caenorhabditis elegans" OR "microalgae" OR aquacultur" OR bivalv" OR deformation OR microplasticity OR nanoplastic* OR nanoparticle\$ OR fatigue OR alloy $\$$ OR steel\$ OR metals\$ OR crystal\$ OR "modeling" OR "modelling" OR spectroscopy OR extraction OR monitoring OR "risk assessment" OR infrared))) to run a topic search in the Web of Science (Thomson Reuters) Core collection database via subscription of the Freie Universität Berlin, Germany. We included any languages, publication years (available timespan: 1945 to 2020) and indexes, but we focused only on articles. We see the peer-review process as a quality measure. Hence, we did not consider screening preprint servers. For more detailed information about the search string development consult the supplementary information. The search yielded 275 records. The records were exported, randomized and their titles screened to identify clear mismatches and potential 
matches (the "level 1 matches"). Studies investigating (1) non-terrestrial systems, (2) toxicity/ physiological reactions in non-soil organisms, (3) microplastic extraction methods, or focusing on (4) nanoplastic, (5) human health effects or reporting (6) morphology, diversity, distribution or abundance of plastic materials in ecosystems (often local case studies) were classified as mismatches. Hence, we excluded 257 records. For the remaining 18 articles, the whole text was screened to identify potential candidate articles that were further investigated according to our inclusion and exclusion criteria. (1) Articles had to present data on soil aggregation (e.g. waterstability, size class weights) and/ or loss or decomposition (i.e. mass loss, rate of substrate degradation) of organic material (e.g. plant litter) for microplastic contaminated samples and associated microplastic-free controls. (2) Only soil based systems were applicable. (3) The microplastic materials $((<5 \mathrm{~mm} ; 8))$ had to be incorporated into the test soil. Foils, mulches or other types of plastic layers on top of the soil surface were not taken into account. Seven articles passed this second screening (the "level 2 matches").

As a second source of articles we checked the citation and reference lists of the matching (level 1 and level 2) articles. Lists of articles citing our identified matches were investigated on the corresponding journal home pages or if this service was not provided via google's "cited by" option. Another three articles were identified and also bibliometrically screened but no further articles were found (Fig. S1).

\section{Data table construction}

The complete search yielded overall 10 articles: eight publications for soil aggregation, one for organic matter loss and another one providing data for both soil aggregation and organic matter loss. With these articles we build three datasets: the (1) soil aggregate formation, (2) stabilization and (3) organic matter loss dataset.

From the matching articles, we extracted data on publication authors and year, the microplastic material comprising information about shape, polymer, concentration applied, but also test system parameters (e.g. sterilization, addition of soil biota) and soil aggregation/ organic matter loss metrics. The data derived from raw data available through supplementary materials, or extracted from figures and tables via web plot digitizer (https://automeris.io/WebPlotDigitizer/; further information on accuracy evaluation depending on data source are given in the supplementary information Table S1). Additionally, we included only experiments without artificial organic matter application or additional stressors (e.g. drought). and we extracted only data from the last harvest day.
Soil aggregation data were presented for wet- or drysieving techniques. For both techniques, the mean weight diameter (MWD) was the dominant metric giving information for aggregate stability for multiple aggregate fractions of decreasing size. For wet-sieving, a test focusing on one soil fraction $<4 \mathrm{~mm}$ was also available (i.e. water-stable aggregates $<4 \mathrm{~mm}[\mathrm{WSA}]$ ). For the soil aggregate stabilization dataset, we preferred MWD over WSA due to higher resolution of aggregate size class contributions. We either used presented MWD metrics or calculated it following the formula: MWD $=\sum_{i=1}^{n} \overline{x_{i}} w_{i}$, where $\overline{x_{i}}$ is the mean diameter of each aggregate size fraction and $w_{i}$ is the proportion of the aggregate mass in each size fraction and the overall sample weight.

For the aggregate formation dataset, we included only data for aggregate fractions larger than the soil used at the beginning of the experiment; i.e. in cases where the soil was sieved to $2 \mathrm{~mm}$ prior to the experiment, we only considered data on aggregate weights in soil fraction $>2$ $\mathrm{mm}$ at the end of the experiment. This metric represents the formation of new macro-aggregates $(>250 \mu \mathrm{m})$. This is due to the particle size of the pre-sieved soil used in the included experiments. The newly formed aggregates are assumed to be built from smaller sized aggregates representing a shift in the aggregate size distribution; a shift from more smaller to more larger aggregates. This fraction was compared to the overall sample weight to calculate the percentage of newly formed aggregates $(\mathrm{NFA}): \mathrm{NFA}=$ size class $>$ initial size $/$ sample weight ${ }_{\text {overall }}$.

For the organic matter loss dataset, we collected data only on decomposition of organic material added to the test system by the experimenter and whose degradation was subsequently assessed. Results of organic matter dynamics (e.g. whole soil or aggregate associated carbon) were not suitable: the applied microplastic itself is a source of carbon, which adds to the soil carbon pool [29] and which is not distinguishable from other carboncontaining organic molecules with standard methods.

\section{Effect sizes}

For the three datasets focusing on soil aggregate formation, stabilization and soil organic matter loss, we calculated specific effect sizes using the natural log response ratio of the treatments and control groups following the function: $\log$ response ratio $=\log \left(\mathrm{X}_{\mathrm{T}} / \mathrm{X}_{\mathrm{C}}\right) . \mathrm{X}_{\mathrm{T}}$ represents the mean data of microplastic treated samples and $\mathrm{X}_{\mathrm{C}}$ the corresponding microplastic-free control means. The effect sizes and the corresponding variances were calculated with the function escalc() in the package "metafor" [30] by integrating treatment and control means, the associated standard deviation (sd) and number of replicates of experimental units $(n)$. In cases where only the 
standard error (se) was reported, we calculated sd as follows: $\operatorname{sd}=\operatorname{sqrt}(\mathrm{n}) \times$ se. One article did not report any metric of variance, here, we used the median of all calculated effect size variances as a surrogate [31].

\section{Subgroup variables}

We collected data on the shape and polymer of the tested microplastics. The variable "shape" comprised fibers, particles and films; particles included all non-linear shapes e.g. irregular shaped fragments (secondary microplastic particles) and regularly shaped beads and spheres. The level "films" represented the addition of microplastic foil and mulch residuals. In the variable polymer we listed all tested microplastic polymers. In one case a mixture of two fibers (polyester and polyacryl) was tested; we included this study and labeled it as "mix" in the variable "polymer".

\section{Statistics}

All statistics were conducted in $\mathrm{R}$ version 4.0.0 [32]. The constructed datasets comprised multiple effect size values per study. To overcome this bias of nonindependent data, we used a two-step approach [33]. First, we excluded factor levels when appropriate. We used in our analysis only the highest microplastic concentration applied in the experiment to detect strongest effects (excluding 36 effect size estimates of 84) and we excluded data on sterile test systems without addition of any soil biota and hence any soil biota activity (excluding 3 effect size estimates of 84). Since data on microplastic effects in plant systems were only available for aggregate stability but not for the other metrics we wanted to investigate, we excluded effect size estimates for presence of plants (removing 9 effect size estimates of 84; see supplementary information Table S2 for the "plant" dataset results).

Second, we calculated weighted means of multiple effect sizes estimates within one study. This was necessary for one publication presenting data on microplastic effects on soil aggregate stability for different fungal strains [34]. We applied a phylogenetically corrected merging by using the $\mathrm{rma} \cdot \mathrm{mv}()$ function in the "metafor" package [30, 35] (merging 5 effect sizes to 1 ). We implemented the phylogenetic information for the fungal strains via the random effect of the multilevel model (for tree construction please refer to [36]). The final dataset comprised 32 observed effect sizes focusing only on test systems without plants (effect sizes on test systems with plants are included in the supplementary data).

Additionally, we identified an authorship dependency issue in our dataset. We followed the procedure by Moulin and Maral [37] to construct author collaboration networks. The bibliometric information of the final matching article list was exported from Web of Science and analyzed in Biblioshiny - the web interface of the R package "bibliometrix" [38]. For the two most recent published matching articles no bibliometric data were available via Web of Science hence author clusters had to be added manually via vector drawing program. The resulting author clusters indicated a strong bias: the dataset consisted mainly of two author clusters (Cluster R: 6 of 10 publications; Cluster Z: 2 of 10 publications; 2 unclustered articles; Fig. S2).

The three final datasets were analyzed by a multilevel (mixed-effects) model with the function rma.mv() in the R package "metafor" [30] implementing study weighting by the inverse of the effect size variance and the restricted maximum likelihood approach. The author cluster information was introduced via a random effect. We ran models without and with inclusion of the subgroup variables shape and polymer. For the test statistics, we chose a t-distribution with $\mathrm{k}$ - $\mathrm{p}$ degrees of freedom (with $\mathrm{k}$ and $\mathrm{p}$ being the number of effect size estimates and model coefficients, respectively, included in the model) mimicking the Knapp and Hartung method [39]. By this, the robustness of the model against false positive results increases [40].

As sensitivity analyses, we applied Eggers's regression test for publication bias [41]. For this regression analysis, the standard error of the effect sizes is implemented into the fixed effect of the multilevel model as numerical variable. By this the effect size precision is tested against its magnitude. If the intercept of the resulting regression line deviates from zero, the analyzed relationship is asymmetrical and hence a publication bias is plausible. The $p$-value for this analysis was set to $p=0.1$ due to small number of observed effect sizes in our analysis and hence reduced statistical power of the applied test statistic [41, 42].The outcomes of these tests can be found in the supplementary information (SI Fig. S3) and all data and references of the articles used for analyses and plotting are available in the supplementary data.

\section{Experiment \\ Experimental design}

We conducted a laboratory experiment in a soil system to test for effects of a diverse set of microplastic polymers representing different shapes. Thus, the experimental design comprised two factors: shape and polymer type. For 'shape' we had four levels and for 'polymer type' we had three levels, resulting in 12 microplastic treatments and a plastic-free control (see Table 1). For each microplastic treatment, we had 10 and for the controls 40 replicates, yielding overall 160 experimental units. 
Table 1 Length and width/ diameter distribution for the used plastic shapes (fibers, films, foams and particles) and the respective polymers: $\mathrm{PES}=$ polyester, $\mathrm{PP}=$ polypropylene, $\mathrm{PA}=$ polyamide, $\mathrm{PET}=$ polyethylene terephthalate, $\mathrm{CPP}=$ casted polypropylene, $\mathrm{PE}=$ polyethylene, $\mathrm{PU}=$ polyurethane, $\mathrm{EPP}=$ expandable polypropylene, $\mathrm{PC}=$ polycarbonate

\begin{tabular}{|c|c|c|c|c|c|c|}
\hline \multirow[t]{2}{*}{ Shape } & \multirow[t]{2}{*}{ Polymer } & \multirow[b]{2}{*}{$\mathbf{N}$} & \multicolumn{2}{|c|}{ Length in $\mathrm{mm}$} & \multicolumn{2}{|c|}{ Width/diameter in $\mathrm{mm}$} \\
\hline & & & mean & sd & mean & sd \\
\hline \multirow[t]{3}{*}{ Fiber } & PES & 100 & 0.899 & 0.736 & 0.030 & 0.004 \\
\hline & $\mathrm{PP}$ & 100 & 1.325 & 0.697 & 0.028 & 0.004 \\
\hline & PA & 100 & 1.248 & 0.794 & 0.030 & 0.006 \\
\hline \multirow[t]{3}{*}{ Film } & CPP & 100 & 5.378 & 1.913 & 4.018 & 1.579 \\
\hline & $P E$ & 100 & 4.478 & 1.087 & 3.208 & 1.623 \\
\hline & PET & 100 & 5.086 & 0.833 & 4.289 & 1.469 \\
\hline \multirow[t]{3}{*}{ Foam } & PU & 100 & 2.858 & 2.203 & 1.691 & 0.975 \\
\hline & $P E$ & 100 & 4.565 & 3.116 & 2.133 & 1.482 \\
\hline & EPP & 100 & - & - & 2.039 & 0.802 \\
\hline \multirow[t]{3}{*}{ Particle } & $\mathrm{PP}$ & 100 & 4.717 & 0.412 & 2.693 & 0.880 \\
\hline & PC & 100 & 2.065 & 1.183 & 1.157 & 0.642 \\
\hline & PET & 100 & 4.939 & 2.401 & 2.621 & 1.098 \\
\hline
\end{tabular}

\section{Microplastic materials}

The plastic treatment consisted of "simulated" secondary microplastic materials (in the sense that they were not produced in the environment), which were processed for this experiment from commercially available products (Fig. 1; for details see SI Table S3). For microfibers, we chose polyamide (nylon), polyester and polypropylene obtained by manually cutting ropes. Microplastic films were produced by a hole puncher and manually cutting polyethylene terephthalate, casted polypropylene and polyethylene foils from supermarkets and gardening supplies. For microplastic foams, we used polyethylene, expandable polypropylene and polyurethane products from packaging and foam rubber padding materials. Microplastic partciles were produced from polycarbonate (CDs/ DVDs), polyethylene terephthalate (water bottles) and polypropylene (planting pots). The microplastic foams and particles were generated via blending (Philips Pro Blend 6 RD). The microfibers and -films could not be obtained by blending due to their high flexibility, not even after freezing the products with liquid nitrogen. It is noteworthy that the factor polymer is confounded by additives since each of the used commercial plastic products comprised its individual combination of colorants and functional additives.

The secondary microplastics varied in their size range but were all $<5 \mathrm{~mm}$, and thus met the definition for microplastics [8] (Table 1). The measurement of the microplastic dimensions was done with Image [43]. The materials were surface sterilized (microwaving for $2 \mathrm{~min}$, 630 watts) before introducing them into the test systems, all at a concentration of $0.4 \%(w: w)$ for dry soil. This concentration was chosen following previous studies demonstrating detrimental microplastic-induced effects on soil aggregation $[18,21]$.

\section{Test system}

We used $6 \mathrm{~cm}$ Petri dishes filled with $15 \mathrm{~g}$ of an unsterilized local soil sieved to $<2 \mathrm{~mm}$ particle size. With this test system, we focused on soil-derived responses related to structural changes and organic matter decomposition. The soil had a sandy loam texture (Albic Luvisol following FAO classification; $74 \%$ sand, $18 \%$ silt, $8 \%$ clay), with $6.9 \mathrm{mg} / 100 \mathrm{~g} \mathrm{P}$ (calcium-acetate-lactate), $5.0 \mathrm{mg} / 100 \mathrm{~g} \mathrm{~K}$ (calcium-acetate-lactate), $0.12 \%$ total $\mathrm{N}$ and $1.87 \%$ total $\mathrm{C}$ content and a soil $\mathrm{pH}$ of 7.1 [44]. The soil for each system was mixed individually with the designated microplastic material, and the controls were mixed with the same energy and for the same time to apply an equivalent disturbance.

In each system, we inserted a miniature tea bag [45] made of $2.5 \times 5 \mathrm{~cm}$ rectangles of $30 \mu \mathrm{m}$ nylon mesh (Sefar Nitex) and an impulse sealer (Mercier Corporation, product no. 127174). The tea bags were filled with $300 \mathrm{mg}$ green tea composed of $49 \%$ C (Lipton green tea, Sencha Exclusive Selection, [45]). The tea had a C:N ratio of 12 , which can be considered as high quality litter that is easy to decompose. The tea bags were small and flat enough to be buried in the soil layer. The test system was initiated by adding autoclaved $\left(121^{\circ} \mathrm{C}\right.$ for $\left.20 \mathrm{~min}\right)$ distilled water to reach a water holding capacity of $60 \%$. Each unit was sealed with parafilm and stored in the dark at $22{ }^{\circ} \mathrm{C}$ for 4 weeks. At harvest, the test systems were opened, dried at $40{ }^{\circ} \mathrm{C}$ and stored until further usage.

\section{Measurements}

We evaluated three system responses to the microplastic contamination. (1) new formed aggregates $>2.0 \mathrm{~mm}$, (2) the percentage of water-stable aggregates and (3) organic matter loss in the miniature tea bags. These represent the three metrics investigated in our meta-analysis on soil aggregate formation, stability and organic matter loss. The soil samples were carefully removed from the Petri dishes and passed through a $4 \mathrm{~mm}$ sieve to break up the dried aggregates, and we retrieved the tea bag.

Subsequently, we tested the samples for the amount of aggregates formed de novo from the initial soil $<2.0 \mathrm{~mm}$ after 4 weeks of incubation [46, 47]. For this, we placed the prepared soil fraction $<4 \mathrm{~mm}$ on a second $2 \mathrm{~mm}$ mesh which we moved vertically two times to allow separation of the soil sample into the two fractions $>2 \mathrm{~mm}$ and $<2 \mathrm{~mm}$ while avoiding abrasion. The amount of newly formed aggregates was then standardized by the overall weight of the sample: 

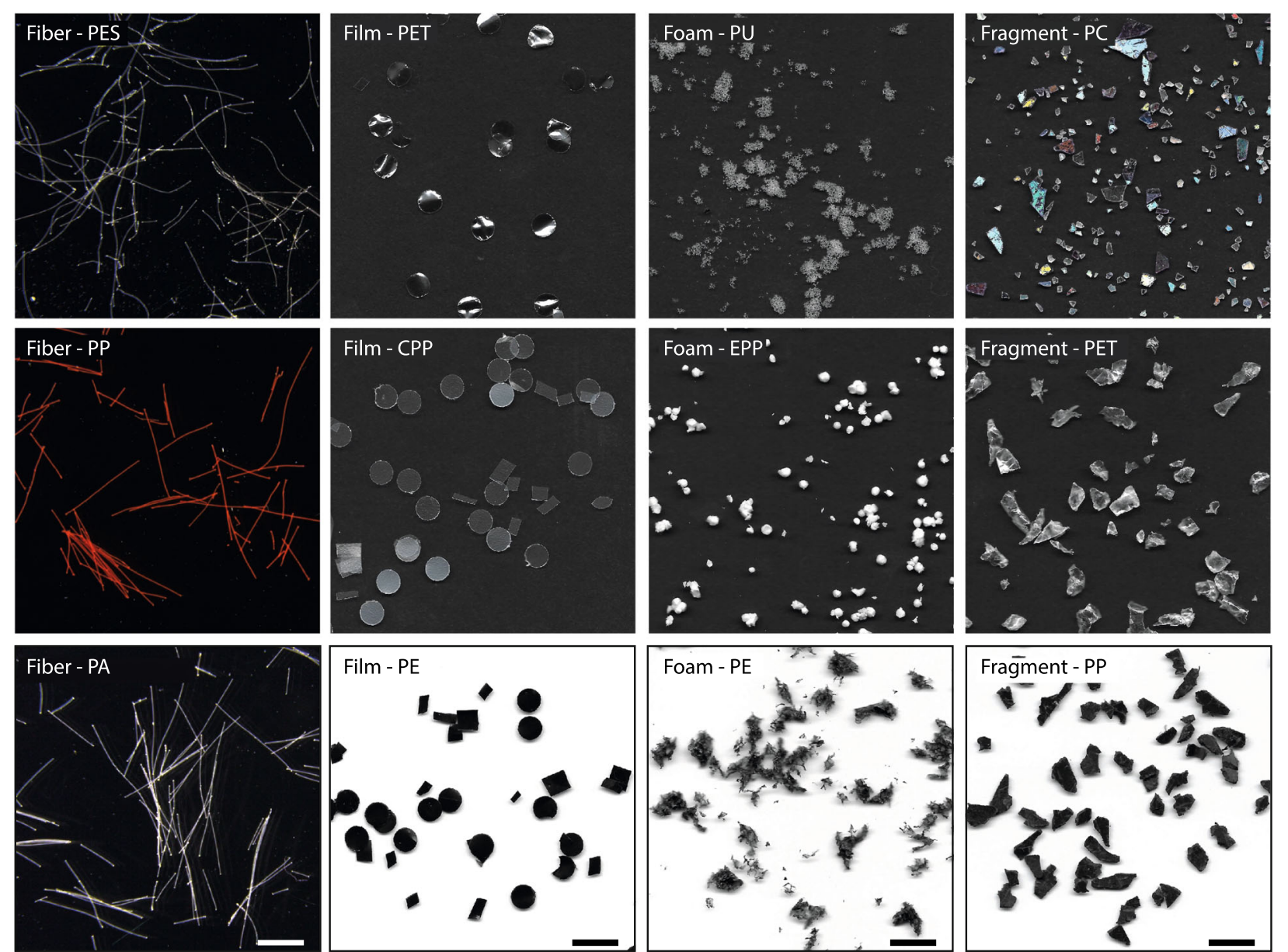

Fig. 1 Overview of used plastic types (fibers, films, foams and particles) and the respective polymers: PES = polyester, PP = polypropylene, PA = polyamide, $\mathrm{PET}=$ polyethylene terephthalate, $\mathrm{CPP}=$ casted polypropylene, $\mathrm{PE}=$ polyethylene, $\mathrm{PU}=$ polyurethane, $\mathrm{EPP}=$ expandable polypropylene, PC = polycarbonate. The white scale bar responds to the fibers only and represents $1 \mathrm{~mm}$ while the black scale bars for films, foams and particles represent $1 \mathrm{~cm}$

Newly formed aggregates $>2 \mathrm{~mm}(\%)=\left(\right.$ fraction $_{>2} \mathrm{~mm}$

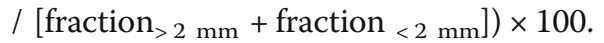

In the next step, we carefully mixed the separated fractions, placed $4.0 \mathrm{~g}$ of each sample on a small sieve with $250 \mu \mathrm{m}$ mesh size, which were allowed to capillarily re-wet with distilled water before inserted into a sieving machine (Agrisearch Equipment, Eijkelkamp, Giesbeek, Netherlands). By this, we measured aggregate stability following a modified protocol by Kemper and Rosenau [23].The machine sieved for $3 \mathrm{~min}$ in water-filled tins filled with distilled water. By this technique, we separated each sample into an unstable fraction, and a water-stable fraction with a size > $250 \mu \mathrm{m}$. The water-stable fraction was further treated to extract sand particles and organic debris. The weight of starting soil volume $(4.0 \mathrm{~g})$ and the waterstable fraction were corrected for this coarse matter fraction.
Percentage of water-stable aggregates $(\%)=[($ water stable fraction - coarse matter) / (4.0 g - coarse matter)] $\times 100$.

Finally, we determined organic matter loss from the miniature tea bags by measuring the relative mass remaining (g g-1) on a dry matter basis. Nine tea bags were lost during the harvest.

\section{Statistics}

All analyses were conducted in R [32]. We tested the effect of our microplastic treatment (three polymers per four shapes) on the three soil-derived responses in a two-step approach. In the first step, we used an estimation method to generate unpaired mean differences (treatment minus control) via bootstrapping (5000 iterations) implemented in the function dabest() in the $\mathrm{R}$ package "dabestr" [48]. The bootstrap estimates were by default bias- and skewness-corrected; hence, we 
generated bias-corrected and accelerated 95\% confidence intervals (CI) [49]. This approach focuses on the magnitude and the precision of an effect further supported by the sampling-error distribution which is also plotted. It allows clear visualization and identification of positive, negative and neutral effects of treatments compared to the controls. If the unpaired mean difference confidence interval (CI) overlaps the zero line (line of no effect) the corresponding effect is neutral, i.e. the treatment caused no detectable response compared to the control.

In the second step, we evaluated our findings by generalized least square models in the "nlme" package [50]. We accounted for heterogeneity in the variables shape and polymer by applying the varIdDent() function. We checked model residuals for heteroskedasticity and normal distribution. For multiple comparison tests of the microplastic treatments against the control samples, we used the glht () function in the package "multcomp" [51] to run a many-to-one comparison procedure [52]. We tested the hypothesis that the mean difference of the microplastic treatments and the controls is $\geq$ zero (treatment - control $\geq 0$ ). Reported $p$-values were adjusted using the single-step method. Model outcomes can be found in the supplementary informations (SI Table S4 and S5) and all data used for analyses and plotting are available in the supplementary data.

\section{Results}

\section{Meta-analysis}

Our meta-analysis provides a quantitative summary of the impact of microplastic soil contamination on the ecosystem processes soil aggregate formation, stabilization and organic matter loss (Fig. 2). We found strong limitations in the amount of available data for the three investigated effect sizes. The majority of studies reported effects of aggregate stability, while only one study presented data on soil organic matter decomposition. Microfibers made of polyester are the most studied microplastic type and polymer across studies, while only three studies tested polyacryl and a textile fiber mix, respectively. Particulate microplastics (e.g. particles, beads, spheres) were targeted in five studies which tested a broad variety of polymers; with one study comparatively testing 6 different plastic polymers.

The magnitude of the three investigated effect sizes was overall neutral (the confidence interval includes zero). This could be ascribed to the limited available data and high variability among effect sizes values within subgroups, causing low statistical power.

\section{Experiment}

We tested 12 different plastic materials, which are a combination of four different shapes (fibers, films, foams and particles) and 3 polymers, for their impact on soil aggregate formation, stabilization and organic matter decomposition.

For the de novo formed aggregates $>2 \mathrm{~mm}$, we found that the presence of fibers consistently reduced the amount of this aggregate fraction irrespective of the plastic polymer (Fig. 3a, SI Fig. S4). For polyethylene and polyethylene terephthalate materials (film - PE, foam PE, film - PET, particle - PET), we detected also a reduction of the amount of new formed aggregates across different plastic shapes. We found the opposite for the polypropylene (PP) materials (film - casted PP, foam expandable PP, particle - PP); for these materials we detected only neutral effects. Polyurethane foam and polycarbonate particles yielded neutral and negative effects, respectively.

For aggregate stability, only 3 microplastic materials caused a detectable effect (Fig. 3b): Polyester fibers and polyethylene foams reduced, while polyethylene films increased aggregate stability. The other materials had neutral effects.

Organic matter loss was the only variable not affected by microfibers (Fig. 3c). Instead, polypropylene films (CPP) and particles and polyethylene films reduced the decomposition of the tea. All other materials had neutral effects.

\section{Discussion}

\section{Meta-analysis}

With this work we present insight into the importance of microplastic shapes for soil ecosystem process components, specifically soil structure and organic matter loss. Our meta-analysis revealed that polyester microfibers are the most often tested plastic materials in experiments focusing on soil responses to microplastic contamination. For this material and shape, negative effects on soil aggregate stability are repeatedly reported $[18,20-22,34]$ but which did not manifest here in this analysis (Fig. 2). For microplastic particles, a range of polymers has been tested, but with no clear pattern emerging for any of the investigated soil responses.

The data collection highlights the lack of a broad experimental base upon which to build mechanistic insights on the impact of microplastic - with its diverse shapes and chemical composition - on major soil ecosystem processes. However, research on microplastic effects on terrestrial systems lags behind those of aquatic systems, hence we can expect a steady increase in new publications on the topic in the near future. Timely updates of these types of data syntheses are thus indispensable.

The focus on fibrous and particulate microplastics also reveals the complete absence of other shapes in the literature. There was no data available on effects of microplastic films or foams on the soil responses we investigated here. Experiments on the effects of film 


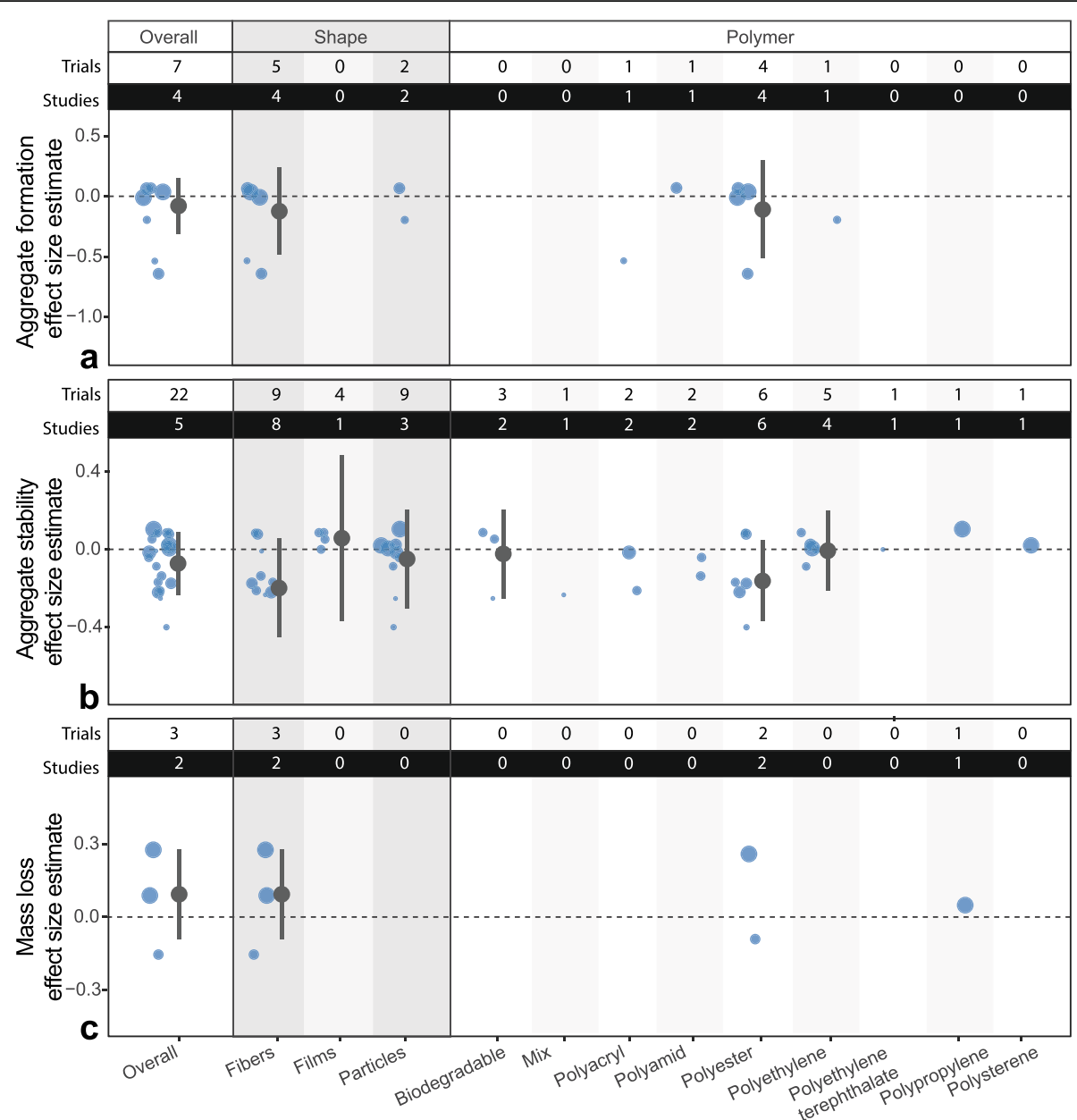

Fig. 2 Overall, microplastic shape and polymer effects on soil aggregation process components and organic matter loss. The distribution of the observed effect sizes is presented as blue-colored dots with circle size depicting study quality; studies with low variability and high number of replicates are represented with large sized symbols. The overall and subgroup effect size estimates and their variances are displayed as means and $95 \%$ confidence intervals for all groups comprising 3 or more observed effect sizes (trials). Additionally, the number of trials (number of observed effect sizes per study) and studies (number of publications giving data to our data sets) contributing to the presented effects are shown on top of each corresponding panel

addition to a terrestrial system exist, but the material is always applied to the soil surface (e.g. [53]), as is done during plastic mulching procedures, or in sizes exceeding the definition of microplastics. Thus, it cannot be evaluated how microplastic films interact with the soil matrix, although they are widely distributed throughout different soils, including natural and agricultural systems [54-58].

A systematic investigation of different shapes is necessary to increase our understanding of the potential consequences of adding new materials with various shapes into soils. To fill this knowledge gap, we experimentally compared the effect of four different microplastic shapes (fibers, films, foams and particles) on soil structure and organic matter loss.

\section{Experiment}

\section{Soil aggregation}

Microfibers reduced the formation of new aggregates > $2 \mathrm{~mm}$ (Fig. 3a). There is no such clear pattern in the available literature, as demonstrated by our metaanalysis (Fig. 2). However, there are experimental studies [20-22] and field observations [59] reporting that microfiber contamination led to reduced concentration of large aggregates. Our findings show clearly that this negative effect on aggregate formation is consistent across microfibers of different chemical composition (i.e. polymer), suggesting that this is indeed an effect primarily related to this linear shape. Observation of microplastic fibers sticking out of aggregates, into which they have apparently been integrated, supports the notion that 

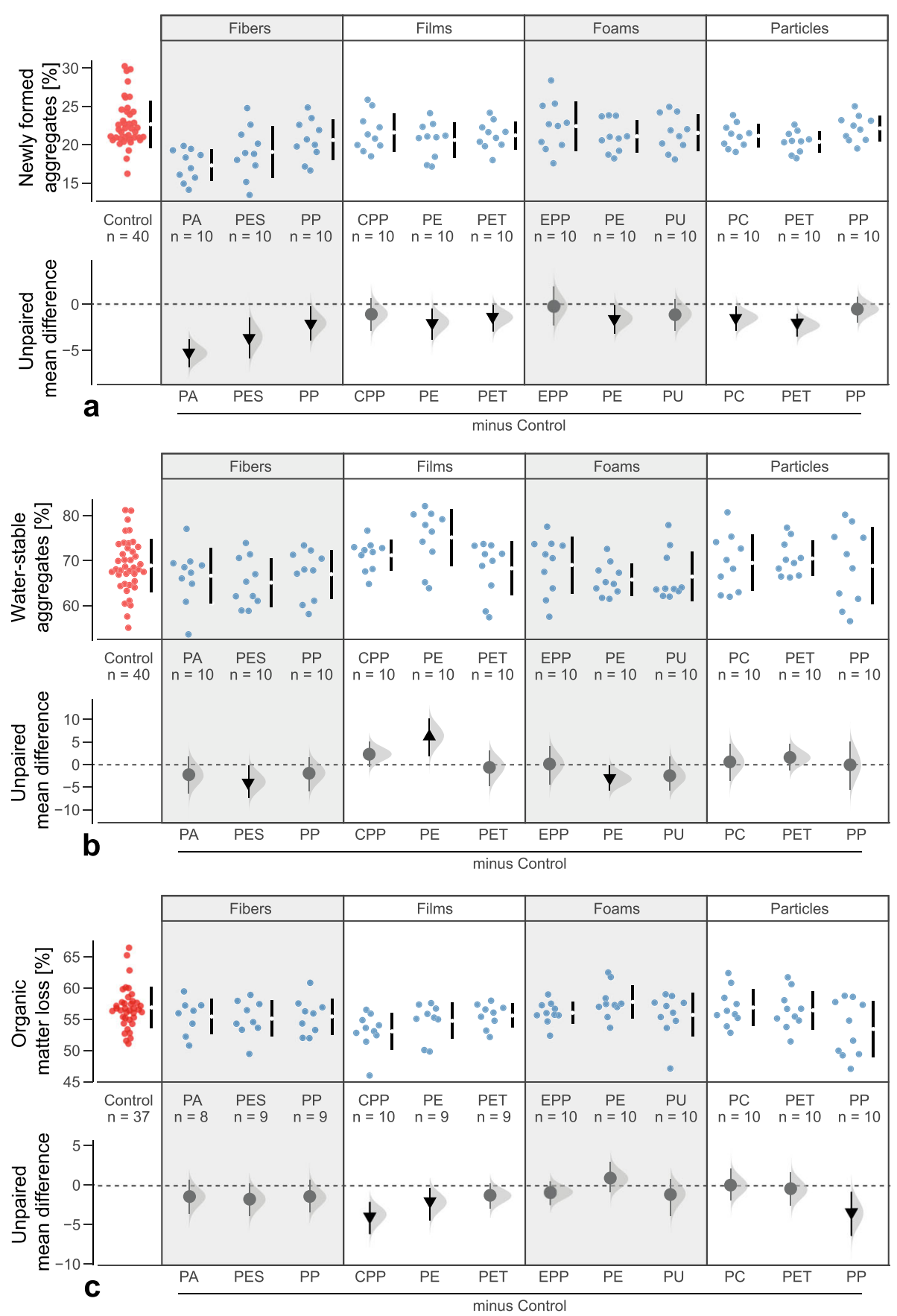

Fig. 3 Microplastic shape and polymer effects on (a) newly formed aggregates $>2 \mathrm{~mm}$ (in \%), (b) stability of aggregates (in \%) and (c) organic matter loss (in \%). Raw data are presented as swarm plots in the first row of each panel and the number of replicates (n) are depicted. The controls are colored in red while microplastic treatments are colored in blue. In the second row of each panel, multi-group estimation plots present the unpaired mean differences of the microplastic treatments and controls. The unpaired means (effect magnitude) are symbolized by circles and triangles and the corresponding confidence intervals (Cls; effect precision) by the vertical, black lines. The sampling error distribution is presented as a grey curve. Negative (arrow head pointing downwards) and positive (arrow head are pointing upwards) effects are colored in black whereas neutral effects (circle) are colored in grey. Outcomes of statistical analysis are presented in SI Table S4 and S5

microfibers introduce fracture points after being incorporated into new formed aggregates (see Fig. 4), likely facilitating breakdown when physical disturbance acts on the aggregate $[19,59]$.
The detrimental effects of microfibers are potentially further modulated by the concentration [18] but also the average dimensions of the material. More trait information about applied microfiber material is needed to 

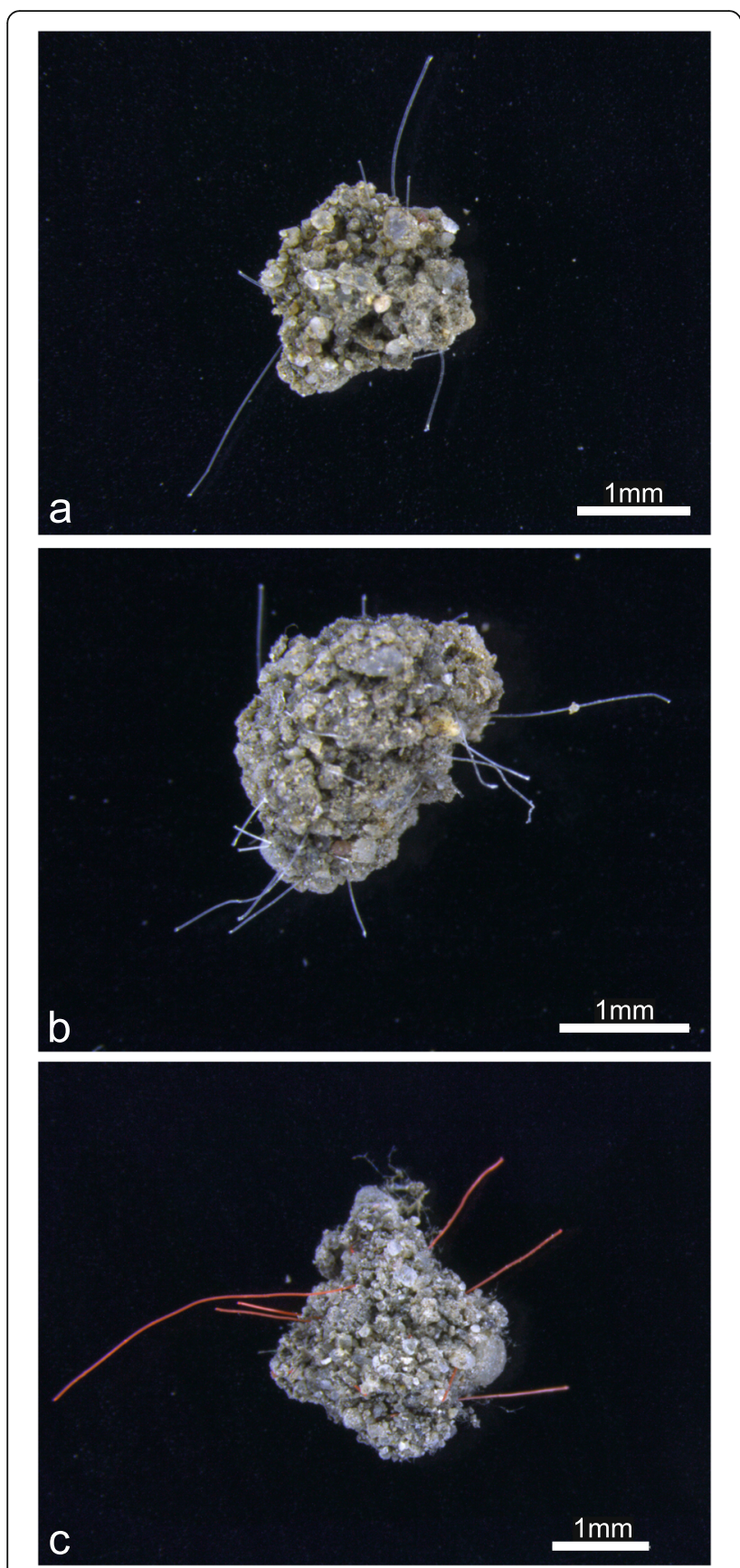

Fig. 4 Photos of soil aggregates which formed during the course of the experiment. Aggregates incorporated microplastic fibers

(polyamide fibers (a), polyester fibers (b), polypropylene fibers $(\mathbf{c})$ )

investigate the potential importance of e.g. curliness, flexibility or surface properties for aggregate formation.

In our experiment, microplastic films negatively affected aggregate formation while positively influencing aggregate stability (Fig. 3a and b). To our knowledge, these are the first data on microplastic film-induced effects on soil aggregation. Previous findings on plastic mulching residuals showed that microplastic films accelerate evaporation, decrease water content, but also reduce the soil tensile strength and bulk density while increasing soil porosity $[60,61]$. This suggests that microplastic films integrated in the soil matrix could introduce artificial pores and breaking planes and thus prevent the formation of large soil aggregates. This would explain the reduced amount of newly formed large macro-aggregates in our experiment. However, the soil aggregates tended to be more stable compared to the controls when incubated with microplastic films. The majority of aggregates in our samples was smaller than $2 \mathrm{~mm}$ while the applied microplastic films had an average dimension of $4.4 \mathrm{~mm}$; thus their size potentially prevented their incorporation into smaller sized aggregates and hence affecting their stability.

We found that microplastic foams tended to decrease the number of newly formed aggregates $>2 \mathrm{~mm}$ and the overall aggregate stability. Foams are clearly underrepresented shapes in microplastic research, although they are a ubiquitous and abundant contaminant, especially in coastal soils [58], due to mariculture, tourism but also usage of insulating, construction, textile and packaging material [62]. As with films, the size will determine on which scale these materials will interact with the soil matrix.

The tested microplastic particles showed a negative effect on the formation of new aggregates $>2 \mathrm{~mm}$ but had no detectable effect on aggregate stability. This negative impact is in line with the literature [18] but was not generally evident as shown by our metaanalysis (Fig. 2). Microplastic particles had a range of soil responses from negative to positive. Particles are less well incorporated into aggregates as compared to fibers [59]; in an agricultural soil, $72 \%$ were soil aggregate-associated while $28 \%$ dispersed in the soil. However, this process is probably determined by the particle size. Other traits like surface smoothness/ roughness, roundness/irregularity, rigidity/flexibility and brittleness/solidity potentially further modulate the induced soil responses.

Considering our results in the context of the shape dissimilarity hypothesis (the more dissimilar an introduced shape (e.g. fiber) is to the natural occurring shapes (e.g. sand grains) the more stronger its effect on the surrounding environment can be) [19], we here found strong evidence that microplastic fibers have a distinct negative impact on soil structure. Other shapes dissimilar to natural occurring shapes did not yield such obvious results. Particles caused comparably detrimental effects as films, while for foams we detected most neutral responses. This may imply that beside shape, the material traits but even more the chemistry is important for the manifestation of microplastic-induced effects on soil structure. 
This is supported by our finding that independently of the shape, polyethylene and polyethylene terephthalate reduced the amount of newly formed aggregates (Fig. 3a). This indicates that these polymers exhibited an inherent chemical property. Plastics can contain a variety of potential toxic substances [63, 64]. Polyethylene can contain several additives like antioxidant agents (e.g. phenolics and phosphites), slip and blocking agents (fatty acids amides) or hydrophilic antistatic agents (e.g. polyethylene glycol esters) of up 0.01-0.5\% weight [65]. Migration of antioxidants and slip agents from low density polyethylene has been widely observed (within hours and days). Polyethylene terephthalate can contain plasticizers, UV-protectors, anti-static agents and postconsumer contaminants like limonene, benzaldehyde, anethole or benzophenone that have been absorbed by the plastic and can migrate from the plastic into the soil matrix $[63,66]$. The quantities of the migrating substances strongly depend on the hydrophobicity of the additive, the initial concentration and the characteristics of the surrounding environment (e.g. water, oil, solvents) $[63,67]$. As demonstrated by Kim et al. [11], leaching of additives can occur in relative short time scales $(24 \mathrm{~h}$ of submerged incubation of microplastics in K-medium) causing detectable toxicity in nematodes reducing their reproduction success. Thus, it can be assumed that in a 4 weeks lasting experiment some additive leaching and migration into soil is possible. To what extent and with which consequences remains to be tested in future experiments. Due to their potential toxicity, the desorption of additives and their transition to the soil can affect soil processes such as microbial activity, subsequently affecting soil aggregation since microbial metabolites (e.g. exo-biopolymers like polysaccharides) can function as gluing substance and promote soil stability [68-70].

It is worth noting that microplastic effects resulting from laboratory studies might underestimate the true microplastic induced impact on the soil matrix compared to a field situation, because the microplastic is not constantly mixed with the soil. In the field, many processes cause constant mixing of microplastics in the soil matrix but also influence the soil aggregation process components formation and stabilization, such as plowing, bioturbation, dry-wet cycles and freeze-thaw cycles, the excretion of plant root exudates and entanglement by arbuscular mycorrhizal fungal hyphae. As a consequence, the microplastic is integrated into soil aggregates more thoroughly and thus the probability for potential impacts of microplastic increases [71].

\section{Organic matter loss}

In our experiment, microplastic films and particles decreased the decomposition of organic matter (here, added as green tea); more specifically, the effects were detectable for casted polypropylene and polyethylene films and polypropylene particles (Fig. 3c). The distinct negative microplastic-induced effects found for soil aggregation did not manifest for organic matter loss. In general, we found no evidence for a shape- but rather a polymer-induced effect.

The literature focusing on microplastic effects on soil organic carbon dynamics are scarce and when available only focusing on microplastic fibers and particles (see Fig. 2c). The research findings so far suggest that microplastic effects on soil organic carbon dynamics are attributed to changes in soil physical parameters (i.e. porosity, connectivity, aeration) [71] and/or sorption and migration of chemicals and additives [72] which in turn affect soil microbial communities, their activity [72-74] and with them the mineralization rates of soil organic matter.

For the polypropylene microplastic film and particles, changes in soil aggregate formation and stabilization could not be ascertained (Fig. 3). Hence it is questionable if additive migrating from these materials might have induced the reductions in organic matter loss. However, additives are commonly detected in terrestrial samples [11, 64] and it can be assumed that their migration potentially has toxic effects on microorganisms [63] leading to impaired soil functions, such as decomposition.

The degradation of organic matter is an important ecosystem function and proxy for soil health that plays a crucial role in nutrient and especially carbon cycling [75]. If some microplastic types could disturb this process, it would have implications for soil C storage. To better understand this potential threat, we need more detailed studies on the influence of different microplastic types and polymers with exact information on their chemical properties.

\section{Conclusions}

Our meta-analysis clearly showed the necessity of shifting the research focus to include non-fibrous microplastic shapes, in particular to overcome the current knowledge gap for microplastic films, foams and particles. Otherwise we run the risk of not having sufficient information for all of these environmentally relevant contaminant shapes. If followed through, this will allow a more formal test of overarching hypotheses about microplastic shape effects.

Our experiment showed a detrimental impact of microplastic fibers on soil aggregate formation and stabilization but not for organic matter loss; the latter variable was affected by other microplastics shapes. This clearly demonstrates that microplastics can differentially impact different ecosystem processes: this is important 
to consider in terms of including various plastic types and a range of response variables in future studies.

There are still many unknowns in the underlying mechanism of microplastic effects; for example, information on polymer properties and additives is rare. More studies like ours, dissecting particular aspects of microplastic diversity, are needed to achieve a more systematic and mechanistic understanding of the effects of these emerging pollutants.

\section{Supplementary Information}

The online version contains supplementary material available at https:/doi. org/10.1186/s43591-021-00007-x

\section{Additional file 1: Figure S1. Flow diagram following PRSIMA which} illustrates the process of data collection and quality control. Figure S2. Author collaboration networks for the 10 publications included in our meta-analysis. The boxes with author names represent the nodes and the connecting lines the links. All co-authors of each publication are depicted and distinct clusters are represented by similar colors. The article collection comprised 2 multi-paper clusters and just 2 unrelated author collaboration clusters. Figure S3. Egger's regression test to evaluate publication bias. Circles represent effect size values from the corresponding aggregate formation, stabilization and organic matter loss datasets plotted against the standard errors (study precision). If the intercept of the resulting regression line deviates from zero, the analyzed relationship is asymmetrically and hence a publication bias is plausible. The $p$-value for this analysis was set to $p=0.1$. No publication bias was evident for the aggregate formation and stability datasets. For the soil organic matter loss dataset, the number of effects size estimates is too low to run a trustworthy publication test. Table S1. Evaluation of data precision depending on the data source for the stability dataset. Table S2. Multilevel model outcomes for the soil aggregate stability effect sizes for test systems including a plant. All effects are neutral because lower and upper borders of the $95 \% \mathrm{Cls}$ include zero. Thus, no negative or positive microplastic-mediated effects are evident in this subset. Table S3. Plastic products used to produce secondary microplastics. Table S4. Outcomes for newly formed aggregates (NFA in $\%, n=70$ ), water-stability of aggregates (WSA in \%, $\mathrm{n}=70$ ) and organic matter loss (OML in $\%, n=61)$ and microplastic shapes. A $p$-value $<0.05$ was considered significant and marked in bold. Table S5. Outcomes for newly formed aggregates (NFA in $\%, n=70$ ), water-stability of aggregates (WSA in $\%, n=70$ ) and organic matter loss (OML in \%, $n=61)$ and microplastic polymer per shape. A pvalue $<0.05$ was considered significant and marked in bold. Figure S4. Microplastic shape effects on (A) newly formed aggregates $>2 \mathrm{~mm}$ (in $\%),(B)$ stability of aggregates (in \%) and (C) organic matter loss (in \%). Raw data are presented as swarm plots in the first row of each panel and number of replicates (n) are depicted. In the second row of each panel, multi-group estimation plots present the unpaired mean differences of the microplastic treatments and controls. The unpaired means (effect magnitude) are symbolized by circles and triangles and the corresponding confidence intervals (Cls; effect precision) by the vertical, black lines. The sampling error distribution is presented as a grey curve. Negative (arrow head down) effects are colored in black whereas neutral effects (circle) are colored in grey. Outcomes of statistical analysis are presented in Table S2 and S3.

Additional file 2.

\section{Acknowledgements}

This manuscript has been released as a Pre-Print at bioRxiv [76].

\section{Authors' contributions}

A.L. designed the study. M.G. set up the experiment; M.G. provided experimental data; A.L. performed the statistical analysis; A.L., E.F.L., and M.C.R. wrote the manuscript; all authors contributed to the final version of the manuscript.

\section{Funding}

MCR acknowledges funding from an ERC Advanced Grant (694368) and the BMBF-funded project $\mu$ Plastic (031B0907A). EFL acknowledges funding from the Deutsche Forschungsgemeinschaft (LE 859/1-1). Open Access funding enabled and organized by Projekt DEAL.

\section{Availability of data and materials}

All data generated or analysed during this study are included in this published article [and its supplementary information files].

\section{Declarations}

\section{Competing interests}

The authors declare no conflict of interest.

Received: 5 November 2020 Accepted: 20 April 2021

Published online: 01 May 2021

\section{References}

1. Cole M, Lindeque P, Halsband C, Galloway TS. Microplastics as contaminants in the marine environment: a review. Mar Pollut Bull. 2011;62(12):2588-97. https://doi.org/10.1016/j.marpolbul.2011.09.025.

2. Eerkes-Medrano D, Thompson RC, Aldridge DC. Microplastics in freshwater systems: a review of the emerging threats, identification of knowledge gaps and prioritisation of research needs. Water Res. 2015;75:63-82. https://doi. org/10.1016/j.watres.2015.02.012.

3. Barnes DKA, Galgani F, Thompson RC, Barlaz M. Accumulation and fragmentation of plastic debris in global environments. Philos Transact Royal Soc B: Biol Sci. 2009;364(1526):1985-98. https://doi.org/10.1098/rstb.2 008.0205 .

4. de Souza Machado AA, Kloas W, Zarfl C, Hempel S, Rillig MC. Microplastics as an emerging threat to terrestrial ecosystems. Glob Chang Biol. 2018;24(4): 1405-16. https://doi.org/10.1111/gcb.14020.

5. Rillig MC, Lehmann A. Microplastic in terrestrial ecosystems. Science. 2020; 368(6498):1430-1. https://doi.org/10.1126/science.abb5979.

6. Browne Mark A, Niven Stewart J, Galloway Tamara S, Rowland Steve J, Thompson RC. Microplastic moves pollutants and additives to Worms, reducing functions linked to health and biodiversity. Curr Biol. 2013;23(23): 2388-92. https://doi.org/10.1016/j.cub.2013.10.012.

7. Wagner M, Scherer C, Alvarez-Muñoz D, Brennholt N, Bourrain X, Buchinger $\mathrm{S}$, et al. Microplastics in freshwater ecosystems: what we know and what we need to know. Environ Sci Eur. 2014;26(1):12. https://doi.org/10.1186/s12302014-0012-7.

8. Hartmann NB, Hüffer T, Thompson RC, Hassellöv M, Verschoor A, Daugaard $A E$, et al. Are we speaking the same language? Recommendations for a definition and categorization framework for plastic debris. Environ Sci Technol. 2019;53(3):1039-47. https://doi.org/10.1021/acs.est.8b05297.

9. Koelmans AA, Bakir A, Burton GA, Janssen CR. Microplastic as a vector for Chemicals in the Aquatic Environment: critical review and model-supported reinterpretation of empirical studies. Environ Sci Technol. 2016;50(7):331526. https://doi.org/10.1021/acs.est.5b06069.

10. Kwon J-H, Chang S, Hong SH, Shim WJ. Microplastics as a vector of hydrophobic contaminants: importance of hydrophobic additives. Integr Environ Assess Manag. 2017;13(3):494-9. https://doi.org/10.1002/ieam.1906.

11. Kim SW, Waldman WR, Kim T-Y, Rillig MC. Effects of different microplastics on nematodes in the soil environment: tracking the extractable additives using an Ecotoxicological approach. Environ Sci Technol. 2020;54(21):1386878. https://doi.org/10.1021/acs.est.0c04641.

12. Allen $S$, Allen $D$, Phoenix VR, Le Roux $G$, Durántez Jiménez $P$, Simonneau $A$, et al. Atmospheric transport and deposition of microplastics in a remote mountain catchment. Nat Geosci. 2019;12(5):339-44. https://doi.org/10.103 8/s41561-019-0335-5.

13. Brahney J, Hallerud M, Heim E, Hahnenberger M, Sukumaran S. Plastic rain in protected areas of the United States. Science. 2020;368(6496):1257-60. https://doi.org/10.1126/science.aaz5819.

14. Steinmetz Z, Wollmann C, Schaefer M, Buchmann C, David J, Tröger J, et al. Plastic mulching in agriculture. Trading short-term agronomic benefits for long-term soil degradation? Sci Total Environ. 2016;550:690-705. https://doi. org/10.1016/j.scitotenv.2016.01.153. 
15. Nizzetto L, Futter M, Langaas S. Are agricultural soils dumps for microplastics of urban origin? Environ Sci Technol. 2016;50(20):10777-9. https://doi.org/10.1021/acs.est.6b04140.

16. Weithmann N, Möller JN, Löder MGJ, Piehl S, Laforsch C, Freitag R. Organic fertilizer as a vehicle for the entry of microplastic into the environment. Sci Adv. 2018:4(4):eaap8060.

17. GESAMP. Sources, fate and effects of microplastics in the marine environment: a global assessment. Exeter: Polestar Wheatons; 2015.

18. Machado AAD, Lau CW, Till J, Kloas W, Lehmann A, Becker R, et al. Impacts of microplastics on the soil biophysical environment. Environ Sci Technol. 2018;52(17):9656-65. https://doi.org/10.1021/acs.est.8b02212.

19. Rillig MC, Lehmann A, Ryo $M$, Bergmann J. Shaping up: toward considering the shape and form of pollutants. Environ Sci Technol. 2019;53(14):7925-6. https://doi.org/10.1021/acs.est.9b03520.

20. Boots B, Russell CW, Green DS. Effects of microplastics in soil ecosystems: above and below ground. Environ Sci Technol. 2019;53(19):11496-506. https://doi.org/10.1021/acs.est.9b03304.

21. Machado AAD, Lau CW, Kloas W, Bergmann J, Bachelier JB, Faltin E, et al. Microplastics can change soil properties and affect plant performance. Environ Sci Technol. 2019;53(10):6044-52. https://doi.org/10.1021/acs.est.9b01339.

22. Zhang GS, Zhang FX, Li XT. Effects of polyester microfibers on soil physical properties: perception from a field and a pot experiment. Sci Total Environ. 2019;670:1-7. https://doi.org/10.1016/j.scitotenv.2019.03.149.

23. Kemper WD, Rosenau RC. Aggregate stability and size distribution. In: Lute A, editor. Methods of soil analysis part I - physical and mineralogical methods. 2nd ed. Madison: SSSA; 1986. p. 425-43.

24. Elliott ET, Coleman DC. Let the soil work for us. Ecol Bull. 1988;39:23-32.

25. Rabot E, Wiesmeier M, Schlüter S, Vogel HJ. Soil structure as an indicator of soil functions: a review. Geoderma. 2018;314:122-37. https://doi.org/10.101 6/j.geoderma.2017.11.009.

26. Horn R, Taubner H, Wuttke M, Baumgartl T. Soil physical properties related to soil structure. Soil Tillage Res. 1994;30(2):187-216. https://doi.org/10.1016/ 0167-1987(94)90005-1.

27. Liu HF, Yang XM, Liu GB, Liang CT, Xue S, Chen H, et al. Response of soil dissolved organic matter to microplastic addition in Chinese loess soil. Chemosphere. 2017;185:907-17. https://doi.org/10.1016/j.chemosphere.2017. 07.064.

28. Moher D, Liberati A, Tetzlaff J, Altman DG. Preferred reporting items for systematic reviews and meta-analyses: the PRISMA statement. BMJ. 2009; 339:b2535. https://doi.org/10.1136/bmj.b2535.

29. Rillig MC. Microplastic disguising as soil carbon storage. Environ Sci Technol. 2018;52(11):6079-80. https://doi.org/10.1021/acs.est.8b02338.

30. Viechtbauer W. Conducting meta-analyses in $\mathrm{R}$ with the metafor package. J Stat Softw. 2010:36(3):48

31. Copas J, Shi JQ. Meta-analysis, funnel plots and sensitivity analysis. Biostatistics. 2000;1(3):247-62. https://doi.org/10.1093/biostatistics/1.3.247.

32. R Development Core Team. R: a language and environment for statistical computing. Version 3.4.1; 2014.

33. Song C, Peacor SD, Osenberg CW, Bence JR. An assessment of statistical methods for nonindependent data in ecological meta-analyses. Ecology. 2000;101(12):e03184. https://doi.org/10.1002/ecy.3184.

34. Liang Y, Lehmann A, Ballhausen M-B, Muller L, Rillig MC. Increasing temperature and microplastic fibers jointly influence soil aggregation by Saprobic fungi. Front Microbiol. 2019;10:2018.

35. Anderson JT. Plant fitness in a rapidly changing world. New Phytol. 2016; 210(1):81-7. https://doi.org/10.1111/nph.13693.

36. Lehmann A, Zheng W, Soutschek K, Roy J, Yurkov AM, Rillig MC. Tradeoffs in hyphal traits determine mycelium architecture in saprobic fungi. Sci Rep. 2019;9(1):14152. https://doi.org/10.1038/s41598-019-50565-7.

37. Moulin TC, Amaral OB. Using collaboration networks to identify authorship dependence in meta-analysis results. Res Synth Methods. 2020;11(5):655-68. https://doi.org/10.1002/jrsm.1430.

38. Aria M, Cuccurullo C. Bibliometrix: an R-tool for comprehensive science mapping analysis. J Informetr. 2017;11(4):959-75. https://doi.org/10.1016/j. joi.2017.08.007.

39. Knapp G, Hartung J. Improved tests for a random effects meta-regression with a single covariate. Stat Med. 2003;22(17):2693-710. https://doi.org/10.1 $002 / \operatorname{sim} .1482$

40. Assink M, Wibbelink CJM. Fitting three-level meta-analytic models in R: a step-by-step tutorial. Quant Methods Psychol. 2016;12(3):154-74. https://doi. org/10.20982/tqmp.12.3.p154
41. Egger M, Smith GD, Schneider M, Minder C. Bias in meta-analysis detected by a simple, graphical test. BMJ. 1997;315(7109):629-34. https://doi.org/1 0.1136/bmj.315.7109.629

42. Habeck CW, Schultz AK. Community-level impacts of white-tailed deer on understorey plants in north American forests: a meta-analysis. AoB Plants. 2015;7. https://doi.org/10.1093/aobpla/plv119.

43. Schneider CA, Rasband WS, Eliceiri KW. NIH image to ImageJ: 25 years of image analysis. Nat Methods. 2012;9(7):671-5. https://doi.org/10.1038/ nmeth.2089.

44. Rillig MC, Mardatin NF, Leifheit EF, Antunes PM. Mycelium of arbuscular mycorrhizal fungi increases soil water repellency and is sufficient to maintain water-stable soil aggregates. Soil Biol Biochem. 2010;42(7):1189-91. https://doi.org/10.1016/j.soilbio.2010.03.027.

45. Keuskamp JA, Dingemans BJJ, Lehtinen T, Sarneel JM, Hefting MM. Tea bag index: a novel approach to collect uniform decomposition data across ecosystems. Methods Ecol Evol. 2013;4(11):1070-5. https://doi.org/10.1111/2 041-210X.12097.

46. Lehmann A, Fitschen K, Rillig MC. Abiotic and biotic factors influencing the effect of microplastic on soil aggregation. Soil Syst. 2019;3(1):21. https://doi. org/10.3390/soilsystems3010021.

47. Lehmann A, Zheng W, Ryo M, Soutschek K, Roy J, Rongstock R, et al. Fungal traits important for soil aggregation. Front Microbiol. 2020;10(2904):2904 https://doi.org/10.3389/fmicb.2019.02904.

48. Ho J, Tumkaya T, Aryal S, Choi H, Claridge-Chang A. Moving beyond $P$ values: data analysis with estimation graphics. Nat Methods. 2019;16(7):5656. https://doi.org/10.1038/s41592-019-0470-3.

49. Efron B. Better bootstrap confidence intervals. J Am Stat Assoc. 1987;82(397): 171-85. https://doi.org/10.1080/01621459.1987.10478410.

50. Pinheiro J, Bates D, DebRoy S, Sarkar D, Team RC. Nlme: linear and nonlinear mixed effects models. R package version 3.1-137; 2018

51. Hothorn T, Bretz F, Westfall P. Simultaneous inference in general parametric models. Biom J. 2008;50(3):346-63. https://doi.org/10.1002/bimj.200810425.

52. Dunnett CW. A multiple comparison procedure for comparing several treatments with a control. J Am Stat Assoc. 1955;50(272):1096-121. https:// doi.org/10.1080/01621459.1955.10501294.

53. Sintim HY, Bandopadhyay S, English ME, Bary Al, DeBruyn JM, Schaeffer SM, et al. Impacts of biodegradable plastic mulches on soil health. Agric Ecosyst Environ. 2019:273:36-49. https://doi.org/10.1016/j.agee.2018.12.002.

54. Brinton WF. Characterization of man-made foreign matter and its presence in multiple size fractions from mixed waste composting. Compost Sci Util. 2005;13(4):274-80. https://doi.org/10.1080/1065657X.2005.10702251.

55. Ding L, Zhang S, Wang X, Yang X, Zhang C, Qi Y, et al. The occurrence and distribution characteristics of microplastics in the agricultural soils of Shaanxi Province, in North-Western China. Sci Total Environ. 2020;720: 137525. https://doi.org/10.1016/.scitotenv.2020.137525.

56. He D, Luo Y, Lu S, Liu M, Song Y, Lei L. Microplastics in soils: analytical methods, pollution characteristics and ecological risks. TrAC Trends Anal Chem. 2018;109:163-72. https://doi.org/10.1016/j.trac.2018.10.006.

57. Qi R, Jones DL, Li Z, Liu Q, Yan C. Behavior of microplastics and plastic film residues in the soil environment: a critical review. Sci Total Environ. 2020; 703:134722. https://doi.org/10.1016/j.scitotenv.2019.134722.

58. Zhou Q, Zhang H, Fu C, Zhou Y, Dai Z, Li Y, et al. The distribution and morphology of microplastics in coastal soils adjacent to the Bohai Sea and the Yellow Sea. Geoderma. 2018;322:201-8. https://doi.org/10.1016/j. geoderma.2018.02.015.

59. Zhang GS, Liu YF. The distribution of microplastics in soil aggregate fractions in southwestern China. Sci Total Environ. 2018;642:12-20. https:// doi.org/10.1016/j.scitotenv.2018.06.004.

60. Jiang XJ, Liu W, Wang E, Zhou T, Xin P. Residual plastic mulch fragments effects on soil physical properties and water flow behavior in the Minqin oasis, northwestern China. Soil Tillage Res. 2017;166:100-7. https://doi.org/10.1016/j.still.2016.10.011.

61. Wan Y, Wu C, Xue Q, Hui X. Effects of plastic contamination on water evaporation and desiccation cracking in soil. Sci Total Environ. 2019;654 576-82. https://doi.org/10.1016/j.scitotenv.2018.11.123.

62. Shah AA, Hasan F, Hameed A, Ahmed S. Biological degradation of plastics: a comprehensive review. Biotechnol Adv. 2008;26(3):246-65. https://doi.org/1 0.1016/j.biotechadv.2007.12.005

63. Hahladakis JN, Velis CA, Weber R, lacovidou E, Purnell P. An overview of chemical additives present in plastics: migration, release, fate and environmental impact during their use, disposal and recycling. J Hazard Mater. 2018;344:179-99. https://doi.org/10.1016/j.jhazmat.2017.10.014. 
64. Wang J, Liu X, Li Y, Powell T, Wang X, Wang G, et al. Microplastics as contaminants in the soil environment: a mini-review. Sci Total Environ. 2019; 691:848-57. https://doi.org/10.1016/j.scitotenv.2019.07.209.

65. Gachter R, Muller H. Plastics additives. 4th ed. Munich: Carl Hanser Verlag; 1993.

66. Widen H, Leufvén A, Nielsen T. Migration of model contaminants from PET bottles: influence of temperature, food simulant and functional barrier. Food Addit Contam. 2004;21(10):993-1006. https://doi.org/10.1080/026520304 00009217.

67. Li B, Wang Z-W, Lin Q-B, Hu C-Y. Study of the migration of stabilizer and plasticizer from polyethylene terephthalate into food simulants. J Chromatogr Sci. 2016;54(6):939-51. https://doi.org/10.1093/chromsci/bmw025.

68. Caesar-Tonthat TC. Soil binding properties of mucilage produced by a basidiomycete fungus in a model system. Mycol Res. 2002;106(8):930-7. https://doi.org/10.1017/S0953756202006330.

69. Dorioz JM, Robert M, Chenu C. The role of roots, fungi and bacteria on clay particle organization. An experimental approach. Geoderma. 1993;56(1):179_ 94. https://doi.org/10.1016/0016-7061(93)90109-X.

70. Tisdall JM, Nelson SE, Wilkinson KG, Smith SE, McKenzie BM. Stabilisation of soil against wind erosion by six saprotrophic fungi. Soil Biol Biochem. 2012; 50:134-41. https://doi.org/10.1016/j.soilbio.2012.02.035.

71. Zhang GS, Zhang FX. Variations in aggregate-associated organic carbon and polyester microfibers resulting from polyester microfibers addition in a clayey soil. Environ Pollut. 2020;258:113716. https://doi.org/10.1016/j. envpol.2019.113716.

72. Wang J, Lv S, Zhang M, Chen G, Zhu T, Zhang S, et al. Effects of plastic film residues on occurrence of phthalates and microbial activity in soils. Chemosphere. 2016;151:171-7. https://doi.org/10.1016/j.chemosphere.2016. 02.076 .

73. Huang Y, Zhao YR, Wang J, Zhang MJ, Jia WQ, Qin X. LDPE microplastic films alter microbial community composition and enzymatic activities in soil. Environ Pollut. 2019;254(Pt A):112983. https://doi.org/10.1016/j.envpol.2 019.112983.

74. Yang X, Bento CPM, Chen H, Zhang H, Xue S, Lwanga EH, et al. Influence of microplastic addition on glyphosate decay and soil microbial activities in Chinese loess soil. Environ Pollut. 2018;242(Pt A):338-47. https://doi.org/10.1 016/j.envpol.2018.07.006

75. Lehmann J, Kleber M. The contentious nature of soil organic matter. Nature. 2015:528(7580):60-8. https://doi.org/10.1038/nature16069.

76. Lehmann A, Leifheit EF, Gerdawischke M, Rillig MC. Microplastics have shape- and polymer-dependent effects on soil processes. bioRxiv. 2020; 2020.06.02.130054:1-24. https://doi.org/10.1101/2020.06.02.130054.

\section{Publisher's Note}

Springer Nature remains neutral with regard to jurisdictional claims in published maps and institutional affiliations.

\section{Submit your manuscript to a SpringerOpen ${ }^{\circ}$ journal and benefit from:}

- Convenient online submission

- Rigorous peer review

- Open access: articles freely available online

- High visibility within the field

- Retaining the copyright to your article

Submit your next manuscript at $\boldsymbol{\nabla}$ springeropen.com 